\title{
Suspended and Bilayer Graphene Growth at Cu Grain Boundaries on Thin Film Cu
}

\author{
Lester F. Lampert and Jun Jiao ${ }^{1}$ \\ 1. Department of Mechanical \& Materials Engineering and Department of Physics, Portland State \\ University, Portland, OR, USA.
}

Although much progress has been made in regards to high-quality, monolayer graphene growth with copper $(\mathrm{Cu})$ foils, $\mathrm{Cu}$ thin film-based graphene growth has seen little improvement largely due to the weak adhesion of $\mathrm{Cu}$ with the typical underlying substrate, $\mathrm{SiO}_{2}$, and related evaporative losses of $\mathrm{Cu}$. Therefore, the best route to high-quality copper thin film-based graphene growth is to optimize with an $\mathrm{SiO}_{2}$ support and either drive the growth process to low temperature with a plasma process or optimize with a thermal growth system bearing in mind high temperature delamination and dewetting issues commonly experience with copper thin films. Here, we report the enhancement of graphene Raman signal intensity due to suspended graphene and bilayer formation at $\mathrm{Cu}$ grain boundaries.

$\mathrm{Cu}$ thin films were deposited onto $\mathrm{SiO}_{2} / \mathrm{Si}$ wafers with $\mathrm{DC}$ sputtering until $1 \mu \mathrm{m}$ thick and then placed directly into a cold-wall growth reactor optimized for graphene growth. Large $\mathrm{Cu}$ grains were achieved at heater temperatures of $780^{\circ} \mathrm{C}$ under $\mathrm{H}_{2}$ flow for 5 mins. Electron backscatter diffraction (EBSD) mapping and scanning electron microscope (SEM) imaging were both performed with a Zeiss Sigma VP SEM equipped with a Nordlys EBSD camera. Optical inspection and Raman spectroscopy/mapping were performed with a Horiba HR800 Raman spectrometer. Raman maps were analysed with GRISP $\mathrm{v} 1.2[1]$.

After growth of graphene, $\mathrm{Cu}$ films formed into grains with a high density of twin boundaries as seen in Fig. 1a. Additionally, large separations between $\mathrm{Cu}$ grains of 0.5-1.0 $\mu \mathrm{m}$ formed (Fig. 1c). Within these separations bilayer graphene forms and stitches together the monolayer graphene highlighted in Fig. 1b. Post-transfer, graphene films crack only at the bilayer formed between $\mathrm{Cu}$ grain boundaries as indicated by optical inspection in Fig. 1d. Graphene nucleation density enhancement has been previously identified by $\mathrm{Han}$ et al. in accordance with $\mathrm{Cu}$ surface morphology [3]. In order to validate the growth of bilayer graphene between $\mathrm{Cu}$ grain boundaries, Raman mapping was performed across several $\mathrm{Cu}$ grain boundaries in Fig. 2a. Interestingly, suspended graphene was identified occasionally at $\mathrm{Cu}$ grain boundaries (Fig. 2b) with $\mathrm{I}_{2 \mathrm{D} / \mathrm{G}}>9$ (Fig. 2e,h) due to reduced doping, resulting in lowered electronelectron scattering [2]. $\mathrm{I}_{\mathrm{D} / \mathrm{G}}$ did not vary with significant spatial dependence as in Fig. 2c. G peak intensity, $\mathrm{I}_{\mathrm{G}}$, is localized to $\mathrm{Cu}$ grain boundaries (Fig. 2d) where $\mathrm{I}_{2 \mathrm{D} / \mathrm{G}}<1$ (Fig. 2b) further indicating bilayer graphene formation at $\mathrm{Cu}$ grain boundaries. The 2D peak FWHM was larger at $\mathrm{Cu}$ grain boundaries indicated by a large relative shift to monolayer graphene as depicted in Fig. 2e. Suspended graphene demonstrated Raman spectra with near-neutral $G$ peak positions having a slight red shift possibly indicating tensile stress present at the bilayer graphene grain boundaries (Fig 2g). [4]

\section{References:}

[1] MG Babenco, L Tao, and D Akinwande, "Graphene Raman Imaging and Spectroscopy Processing v1.2” https://nanohub.org/resources/grisp. (2014).

[2] S Berciaud, S Ryu, and TF Heinze, Nano Letters 9 (2009), p.346.

[3] GH Han, et al Nano Letters 11 (2011), p.4144. 
[4] The authors acknowledge funding from the National Science Foundation, Intel Corp., and the Oregon Nanoscience and Microtechnologies Institute.
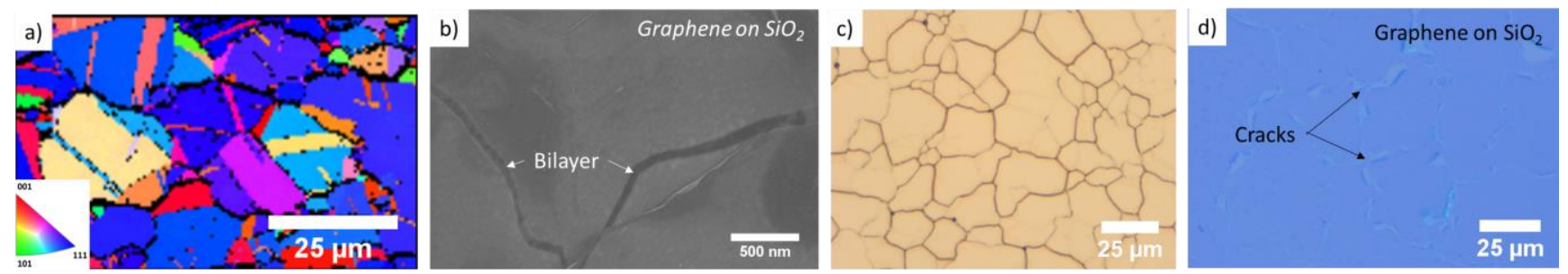

Figure 1. a) SEM EBSD inverse pole figure for $\mathrm{Cu}$ cubic orientation out of the sample plane (z), b) SEM image of the graphene transferred to $\mathrm{SiO}_{2}$ demonstrating bilayer formation at $\mathrm{Cu}$ grain boundaries, c) optical brightfield image of as-grown graphene supported by $\mathrm{Cu} / \mathrm{SiO}_{2} / \mathrm{Si}$, d) optical brightfield image of graphene transferred to $\mathrm{SiO}_{2}$ with cracks forming at weak bilayer grain boundaries.
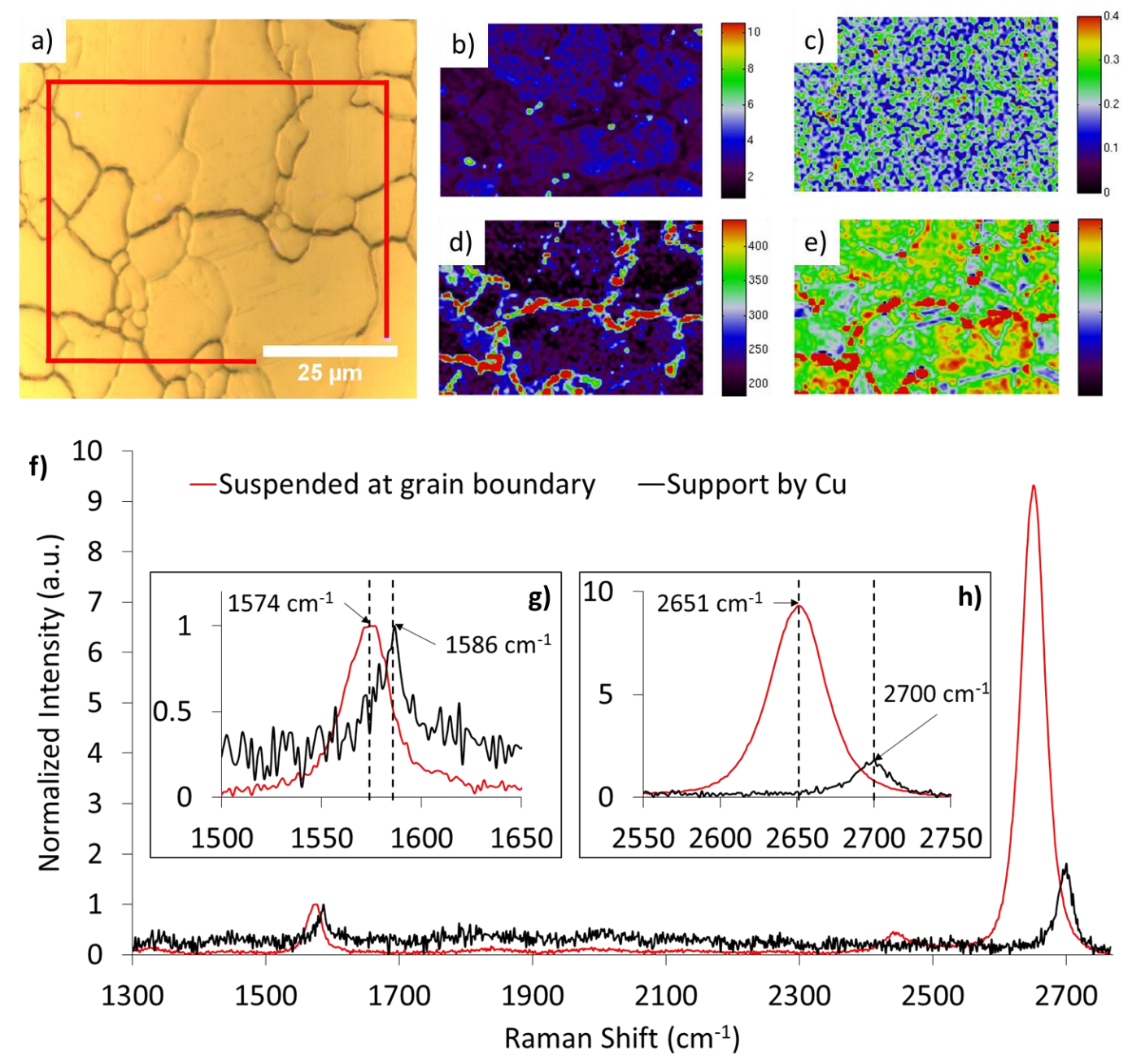

Figure 2. a) Optical brightfield image with region of interest marked for Raman maps, b-e) Raman maps for $\mathrm{I}_{2 \mathrm{D} / \mathrm{G}}, \mathrm{I}_{\mathrm{D} / \mathrm{G}}, \mathrm{I}_{\mathrm{G}}$, and 2D relative FWHM, respectively, f) Raman spectra for suspended and Cu-supported graphene, g) G peak inset depicting relative shift, h) 2D peak inset depicting relative shift and enhancement of suspended graphene. 